\author{
SoTEL Symposium 19-20 February 2020
}

\title{
Title: Using Video in Blended Pedagogies to Address Accessibility
}

\author{
Grant Beevers \\ Kultura \\ grant.beevers@kaltura.com \\ Submission Type: Brief Presentation (20mins + 10mins Q\&A) \\ Keywords: disability, accessibility, video
}

\begin{abstract}
:
Besides being the right thing to do, creating an accessible learning experience ensures that educational institutions are providing equitable opportunities for the many students with a disability. Millions of people live with disabilities, not all of them easily visible. Video has become a major component of the digital workplace, and offers some challenges to some people with disabilities.
\end{abstract}

But not only are there ways to overcome these challenges, video itself can aid in providing an effective learning experience for all students. The most common topic to come up for increasing video accessibility is around captions and transcripts. Video unites sight and sound. Especially in an education environment, removing the sound usually makes a video mostly pointless. It's not enough for the content to be accessible, though. The video platform itself has to be accessible if you genuinely want to increase accessibility.

The key to increasing accessibility is flexibility. If you can offer students multiple choices with captions or without, with default-size icons or with larger and higher contrast icons, live or on-demand, in person or remote - they can choose for themselves the accommodations they need to do their best work.

Many students have disabilities they may not wish to disclose. The way one person is affected by a disability may be different from how another person with a similar disability is affected. In fact, a person may feel impacted by their disability in different ways from day to day, such as when someone with chronic pain has a "good" or a "bad" day.

In this brief presentation, Grant Beevers, Senior Digital Learning Specialist with Kaltura, will outline how organisations can address accessibility through best practice in video design and publishing, whether it's embedded video content in course design, content imported from external websites, and common use cases including personal and lecture capture. 